\title{
Front Matter: Volume 9601
}

, "Front Matter: Volume 9601," Proc. SPIE 9601, UV, X-Ray, and Gamma-Ray Space Instrumentation for Astronomy XIX, 960101 (29 September 2015); doi: $10.1117 / 12.2217876$

SPIE Event: SPIE Optical Engineering + Applications, 2015, San Diego, California, SPIE. United States 


\section{PROCEEDINGS OF SPIE}

\section{UV, X-Ray, and Gamma-Ray Space Instrumentation for Astronomy XIX}

\section{Oswald H. W. Siegmund}

Editor

9-10 August 2015

San Diego, California, United States

Sponsored and Published by

SPIE 
The papers in this volume were part of the technical conference cited on the cover and title page. Papers were selected and subject to review by the editors and conference program committee. Some conference presentations may not be available for publication. Additional papers and presentation recordings may be available online in the SPIE Digital Library at SPIEDigitallibrary.org.

The papers reflect the work and thoughts of the authors and are published herein as submitted. The publisher is not responsible for the validity of the information or for any outcomes resulting from reliance thereon.

Please use the following format to cite material from these proceedings:

Author(s), "Title of Paper," in UV, X-Ray, and Gamma-Ray Space Instrumentation for Astronomy $X I X$, edited by Oswald H. Siegmund, Proceedings of SPIE Vol. 9601 (SPIE, Bellingham, WA, 2015) Sixdigit Article CID Number.

ISSN: 0277-786X

ISSN:1996-756X (electronic)

ISBN: 9781628417678

Published by

SPIE

P.O. Box 10, Bellingham, Washington 98227-0010 USA

Telephone +1 3606763290 (Pacific Time) · Fax +1 3606471445

SPIE.org

Copyright (C) 2015, Society of Photo-Optical Instrumentation Engineers.

Copying of material in this book for internal or personal use, or for the internal or personal use of specific clients, beyond the fair use provisions granted by the U.S. Copyright Law is authorized by SPIE subject to payment of copying fees. The Transactional Reporting Service base fee for this volume is $\$ 18.00$ per article (or portion thereof), which should be paid directly to the Copyright Clearance Center (CCC), 222 Rosewood Drive, Danvers, MA 01923. Payment may also be made electronically through CCC Online at copyright.com. Other copying for republication, resale, advertising or promotion, or any form of systematic or multiple reproduction of any material in this book is prohibited except with permission in writing from the publisher. The CCC fee code is $0277-786 \mathrm{X} / 15 / \$ 18.00$.

Printed in the United States of America.

Publication of record for individual papers is online in the SPIE Digital Library.

\section{SPIE. DIGITAL}

Paper Numbering: Proceedings of SPIE follow an e-First publication model. A unique citation identifier (CID) number is assigned to each article at the time of publication. Utilization of CIDs allows articles to be fully citable as soon as they are published online, and connects the same identifier to all online and print versions of the publication. SPIE uses a six-digit CID article numbering system structured as follows:

- The first four digits correspond to the SPIE volume number.

- The last two digits indicate publication order within the volume using a Base 36 numbering system employing both numerals and letters. These two-number sets start with 00, 01, 02, 03, 04, $05,06,07,08,09,0 A, O B \ldots$. OZ, followed by 10-1Z, 20-2Z, etc. The CID Number appears on each page of the manuscript. 


\title{
Contents
}

\author{
$\checkmark \quad$ Authors \\ ix Conference Committee
}

\section{SESSION $1 \quad$ X-RAY TECHNOLOGIES AND MISSIONS I}

960103 CdTe focal plane detector for hard x-ray focusing optics [9601-2]

960104 First results from the OGRESS sounding rocket payload [9601-3]

960105 Technological developments of the OGRE focal plane array [9601-4]

960106 A primer for telemetry interfacing in accordance with NASA standards using low cost FPGAs [9601-5]

\section{SESSION $2 \quad$ X-RAY TECHNOLOGIES AND MISSIONS II}

960107 Modeling contamination migration on the Chandra X-ray Observatory: III [9601-6]

960109 The optical blocking filter for the ATHENA wide field imager: ongoing activities towards the conceptual design [9601-8]

9601 OA Development of the strontium iodide coded aperture (SICA) instrument [9601-9]

\section{SESSION 3 SOLID STATE DETECTORS}

9601 OB Recent progress and development of a speedster-EXD: a new event-triggered hybrid CMOS x-ray detector [9601-10]

9601 OE The Soft X-ray Imager (SXI) for the ASTRO-H Mission [9601-13]

9601 OF Initial results from a cryogenic proton irradiation of a p-channel CCD [9601-14]

\section{SESSION 4 SPACEBORNE X-RAY EXPERIMENTS}

$9601 \mathrm{OH}$ Development of the wide field imager for Athena [9601-16]

9601 Ol LAMP: a micro-satellite based soft $x$-ray polarimeter for astrophysics [9601-17]

$96010 J$ The X-ray Surveyor Mission: a concept study [9601-18]

9601 OL ATHENA: system design and implementation for a next generation $x$-ray telescope [9601-34] 
9601 ON Detector performance for the FIREBall-2 UV experiment [9601-21]

960100 Noise and dark performance for FIREBall-2 EMCCD delta-doped CCD detector [9601-22]

9601 OP Solar glint suppression in compact planetary ultraviolet spectrographs [9601-23]

$96010 Q \quad$ Characterizing, controlling, and correcting distortions in the COS FUV detector [9601-24]

SESSION $6 \quad$ UV TECHNOLOGIES AND MISSIONS II

9601 OR New UV instrumentation enabled by enhanced broadband reflectivity lithium fluoride coatings [9601-25]

9601 OS Characterization of borosilicate microchannel plates functionalized by atomic layer deposition [9601-26]

9601 OT Performance results of the ICON FUV sealed tube converters [9601-27]

POSTER SESSION

9601 OV A transmissive x-ray polarimeter design for hard x-ray focusing telescopes [9601-29]

9601 OW Characterization of Al0.8Ga0.2As geiger photodiode [9601-30]

$96010 X \quad$ Current progress in the characterization of atomic layer deposited $\mathrm{AlF}_{3}$ for future astronomical ultraviolet mirror coatings [9601-31]

9601 OY Silicon photomultipliers for next generation high-energy space telescopes [9601-32]

$96010 Z$ VUV testing of science cameras at MSFC: QE measurement of the CLASP flight cameras [9601-33]

960110 The UV multi-object slit-spectrograph FIREBall-2 simulator [9601-35] 


\title{
Authors
}

Numbers in the index correspond to the last two digits of the six-digit citation identifier (CID) article numbering system used in Proceedings of SPIE. The first four digits reflect the volume number. Base 36 numbering is employed for the last two digits and indicates the order of articles within the volume. Numbers start with 00, 01, 02, 03, 04, 05, 06, 07, 08, 09, OA, OB...0Z, followed by 10-12, 20-2Z, etc.

\author{
Anabuki, Naohisa, OE \\ Ayre, M., OL \\ Baldini, Luca, Ol \\ Bamba, Aya, OE \\ Bandler, Simon R., OJ \\ Barbera, Marco, 09, OH \\ Bautz, Marshall W., OJ \\ Bavdaz, M., OL \\ Beabout, B., $0 Z$ \\ Beabout, D., $0 Z$ \\ Becker, George D., OQ \\ Bellazzini, Ronaldo, Ol \\ Brand, Thorsten, $\mathrm{OH}$ \\ Branduardi-Raymont, G., 09 \\ Brez, Alessandro, 01 \\ Burrows, David N., OB, OJ \\ Burt, D., OF \\ Campbell, Joe C., OW \\ Carver, Alexander, ON \\ Champey, P., OZ \\ Chen, X. J., OW \\ Chen, Yaojia, OW \\ Cheng, Samuel, ON \\ Christe, Steven, 03 \\ Christian, James F., OW \\ Cirtain, J., $\mathrm{OZ}$ \\ Colebrook, David, 05 \\ Collura, A., 09 \\ Comastri, A., 09 \\ Cook, Jason C., OP \\ Cordes, Ryan, OA \\ Corlies, L., 10 \\ Craven, C. A., OS \\ Curtis, T., OT \\ Dahmer, Matthew T., 07 \\ Darling, N. T., OT \\ Davis, Michael W., OP \\ Debes, John H., OQ \\ Del Hoyo, Javier, OR \\ Delaigue, S., OY \\ Deng, Zhi, OV \\ DeRoo, Casey, 05 \\ Dong, Yongwei, OV \\ Dotani, Tadayasu, OE \\ Doty, John P., OE \\ Dryer, B., OF \\ Eder, Josef, 09, $\mathrm{OH}$ \\ Ely, Justin, $O Q$ \\ Ertley, C., OS
}

\author{
Falcone, Abraham D., OB, OJ \\ Farn, David, 05 \\ Feng, Hua, OI, OV \\ Ferreira, I., OL \\ Fleming, Brian T., OR \\ France, Kevin, OR, OX \\ Fürmetz, Maria, $\mathrm{OH}$ \\ Galliano, M., OY \\ Gaskin, Jessica A., 03, 0J \\ Gladstone, G. Randall, OP \\ Goodsall, Timothy, ON \\ Gow, J.P. D., OF \\ Grange, R., 10 \\ Grant, Catherine E., 07 \\ Grava, Cesare, OP \\ Greathouse, Thomas K., OP \\ Gregory, Kyle, 03 \\ Griffith, Christopher V., OB \\ Grove, J. Eric, OA \\ Hall, D. J., OF \\ Hamden, Erika T., ON, 00 \\ Han, Dong, OV \\ Harrison, Fiona A., OJ \\ Hatsukade, Isamu, OE \\ Hayashida, Kiyoshi, OE \\ $\mathrm{He}$, Li, OV \\ Heilmann, Ralf K., OJ \\ Heinz, Sebastian, OJ \\ Heng, Peiying, OV \\ Hennessy, John, ON, OX \\ Hiraga, Junko S., OE \\ Hoadley, Keri, OR \\ Hoenk, Michael, ON \\ Holland, Andrew D., 05, OF \\ Holland, Karen, 05 \\ Hopkins, Randall C., OJ \\ Houret, B., OY \\ Hyde, D., $\mathrm{OZ}$ \\ Inglis, Andrew, 03 \\ Jelinsky, S., OT \\ Jewell, April D., ON, OX \\ $\mathrm{Ji}$, Jianfeng, OV \\ Johnson, E. B., OW \\ Jones, Todd, ON \\ Kamisiński, T., 09 \\ Kersgaard, Eliot, OX \\ Kilbourne, Caroline A., OJ \\ Kimura, Masashi, OE \\ Knödlseder, J., OY
}


Kobayashi, K., $\mathrm{OZ}$

Kohmura, Takayoshi, OE

Kouveliotou, Chryssa, 0J

Kraft, Ralph P., OJ

Kravtsov, Andrey V., OJ

Kruczek, Nicholas, OR

Kyne, Gillian, 00

Lacombe, K., OY

Li, Hong, Ol, OV

$\mathrm{Li}$, Tenglin, OV

Linder, M., OL

Lingner, Nicole, 00

Liu, Yinong, OV

Lo Cicero, U., 09

Lockwood, Sean A., OQ

Lu, Fangjun, OV

Lumb, D., OL

Mane, A. U., OS

Marshall, Herman L., 07

Martin, D. Christopher, ON, 00

Massa, Derck, $0 Q$

McCoy, Jake, 04, 06

McEntaffer, Randall L., 04, 05, 06, 0J

McPhate, J., OT

Mège, P., 10

Meidinger, Norbert, 09, $\mathrm{OH}$

Miles, Drew, 04, 06

Milliard, B., 10

Mineo, T., 09

Minot, M. J., OS

Minuti, Massimo, 01

Mitchell, Lee J., OA

Molendi, S., 09

Moore, Christopher Samuel, OX

Mori, Koji, OE

Morrissey, Patrick, 00

Muleri, Fabio, 0 I

Murakami, Hiroshi, OE

Murray, Neil J., 05, OF

Nagino, Ryo, OE

Nakajima, Hiroshi, OE

Nandra, Kirpal, $\mathrm{OH}$

Natarajan, Priyamvada, OJ

Natsukari, Chikara, OE

Nikzad, Shouleh, ON, OX

Nishioka, Yusuke, OE

Nobukawa, Masayoshi, OE

O'Dell, Stephen L., 07, OJ

Oliveira, Cristina M., $0 Q$

O'Mahony, A., OS

Ong, Hwei Ru, ON

Ozaki, Masanobu, OE

Panessa, Marco, 03

Pareschi, Giovanni, Ol

Parodi, G., 09

Pascal, S., 10

Penton, Steven V., OQ

Petre, Robert, 0J

Phlips, Bernard F., OA
Pietschner, Daniel, $\mathrm{OH}$

Pilch, A., 09

Pinchera, Michele, 01

Piro, L., 09

Plattner, Markus, $\mathrm{OH}$

Plucinsky, Paul P., 07

Popecki, M., OS

Prieskorn, Zachary R., OB, OJ

Proffitt, Charles, $0 Q$

Ptak, Andrew F., OJ

Quijada, Manuel A., OR

Quiret, S., 10

Ramon, P., OY

Ramsey, Brian D., OJ

Rataj, M., 09

Rau, Arne, $\mathrm{OH}$

Rauw, G., 09

Reid, Paul B., OJ

Reiffers, Jonas, $\mathrm{OH}$

Ren, Min, OW

Retherford, Kurt D., OP

Robertson, B., $0 Z$

Rogers, Thomas, 04, 05, 06

Roman-Duval, Julia, $0 Q$

Rouaix, G., OY

Sahnow, David J., OQ

Salmaso, Bianca, 01

Sana, Hugues, OQ

Schiminovich, David, ON

Schneider, Andreas, 03

Schnell, Andrew R., OJ

Schultz, Ted, 04, 05, 06

Schwartz, Daniel A., OJ

Schwarz, J., OS

Sciortino, L., 09

Sciortino, S., 09

Seller, Paul, 03

Sgrò, Carmelo, 0 I

Shapiro, Charles, ON

She, Rui, 01

Shen, Zhengxiang, 01

Shih, Albert Y., 03

Siegmund, O. H. W., OS, OT

Soffitta, Paolo, 0 I

Sonnentrucker, Paule, $0 Q$

Spandre, Gloria, 이

Spiga, Daniele, 0 I

Stewart, M., $\mathrm{OZ}$

Strecker, Rafael, $\mathrm{OH}$

Swartz, Douglas A., 07

Tagliaferri, Gianpiero, 0 I

Tanaka, Takaaki, OE

Tananbaum, Harvey D., OJ

Tayabaly, Kashmira, 0 I

Taylor, Joanna, $0 Q$

Tedesco, J., OT

Tennant, Allyn F., 07

Tice, Neil W., 07 
Tomida, Hiroshi, OE

Townsley, Leisa K., OJ

Tsunemi, Hiroshi, OE

Tsuru, Takeshi G., OE

Tutt, James H., 04, 05, 06

Uchida, Hiroyuki, OE

Ueda, Shutaro, OE

Veale, Matthew C., 03

Vibert, D., 10

Vikhlinin, Alexey A., 07, 0J

Virmontois, C., OY

Wang, Zhanshan, 0 I

Wawer, P., 09

Weatherill, Daniel, 05

Weisskopf, Martin C., OJ

Wen, Mingwu, Ol

Wille, E., OL

Wilms, Jörn, $\mathrm{OH}$

Wilson, Matthew D., 03

Wilson-Hodge, Colleen, 03

Winebarger, A., OZ

Wood, D., OF

Wu, Qiong, OV

$\mathrm{Xu}$, Renxin, $\mathrm{O}$

Yamauchi, Makoto, OE

Zeng, Ming, OV

Zhan, Yafeng, 0

Zhang, Shuangnan, OV

Proc. of SPIE Vol. $9601960101-7$ 
Proc. of SPIE Vol. $9601960101-8$

Downloaded From: https://www.spiedigitallibrary.org/conference-proceedings-of-spie on 26 Apr 2023 Terms of Use: https://www.spiedigitallibrary.org/terms-of-use 


\section{Conference Committee}

Program Track and Conference Chair

Oswald H. W. Siegmund, University of California, Berkeley

(United States)

Conference Program Committee

Stephan R. McCandliss, Johns Hopkins University (United States)

Camden D. Ertley, University of California, Berkeley (United States)

Brian T. Fleming, University of Colorado at Boulder (United States)

James C. Green, University of Colorado at Boulder (United States)

Anton Tremsin, University of California, Berkeley (United States)

Session Chairs

1 X-Ray Technologies and Missions I

Camden D. Ertley, University of California, Berkeley (United States)

2 X-Ray Technologies and Missions II

Brian T. Fleming, University of Colorado at Boulder (United States)

3 Solid State Detectors

Oswald H. W. Siegmund, University of California, Berkeley (United States)

4 Spaceborne X-Ray Experiments

Camden D. Ertley, University of California, Berkeley (United States)

5 UV Technologies and Missions I

Matthew Beasley, Planetary Resources, Inc. (United States)

6 UV Technologies and Missions II

Brian T. Fleming, University of Colorado at Boulder (United States) 
Proc. of SPIE Vol. $9601960101-10$

Downloaded From: https://www.spiedigitallibrary.org/conference-proceedings-of-spie on 26 Apr 2023 Terms of Use: https://www.spiedigitallibrary.org/terms-of-use 